\title{
Effects of Signal Peptide and Adenylate on the Oligomerization and Membrane Binding of Soluble SecA
}

\author{
Ji-Yeun Shin', Mihee $\mathrm{Kim}^{2}$ and Taeho $\mathrm{Ahn}^{2, *}$ \\ ${ }^{1}$ Department of Molecular Endocrinology, Chonnam National University, Gwangju 500-757, Korea \\ ${ }^{2}$ Department of Biochemistry, College of Veterinary Medicine, Chonnam National University, Gwangju 500-757, Korea
}

Received 30 December 2005, Accepted 17 March 2006

\begin{abstract}
SecA protein, a cytoplasmic ATPase, plays a central role in the secretion of signal peptide-containing proteins. Here, we examined effects of signal peptide and ATP on the oligomerization, conformational change, and membrane binding of SecA. The wild-type (WT) signal peptide from the ribose-binding protein inhibited ATP binding to soluble SecA and stimulated release of ATP already bound to the protein. The signal peptide enhanced the oligomerization of soluble SecA, while ATP induced dissociation of SecA oligomer. Analysis of SecA unfolding with urea or heat revealed that the WT signal peptide induces an open conformation of soluble SecA, while ATP increased the compactness of SecA. We further obtained evidences that the signal peptide-induced oligomerization and the formation of open structure enhance the membrane binding of SecA, whereas ATP inhibits the interaction of soluble SecA with membranes. On the other hand, the complex of membrane-bound SecA and signal peptide was shown to resume nucleotide-binding activity. From these results, we propose that the translocation components affect the degree of oligomerization of soluble SecA, thereby modulating the membrane binding of SecA in early translocation pathway. A possible sequential interaction of SecA with signal peptide, ATP, and cytoplasmic membrane is discussed.
\end{abstract}

Keywords: Membrane, Nucleotide, Oligomerization, SecA, Signal peptide

\section{Introduction}

SecA is a central component of the translocation machinery for newly synthesized secretory proteins of Escherichia coli (E. coli). The protein is equally distributed in vivo between the inner membrane and cytosol (Cabelli et al., 1991) forming a protein-conducting channel with SecYEG complex in the membrane (Manting, 2000). During the translocation, SecA interacts directly with various molecules involved (Mori and Ito, 2001) in order to ensure its efficiency (Lill et al., 1989; Brundage et al., 1990). As an ATPase, SecA provides the driving force for the secretion of preprotein across cytoplasmic membrane. The ATPase activity is increased by interaction with translocation machineries such as anionic phospholipid, preprotein, and SecYEG (Lill et al., 1989; Douville et al., 1995). However, different experiments led to contradictory results regarding the sequential binding of ATP and its role in the interaction of SecA with membranes; some studies indicate that ATP inhibits SecA binding and insertion into model membrane (Breukink et al., 1992; Keller et al., 1995), but others suggest that ATP binding promotes SecA interaction with membranes (Economou and Wickner, 1994; Economou et al., 1995). Furthermore, controversies exist over the stepwise interaction of SecA with secretory components during translocation.

The SecA protein displays an unusual physical property in that equilibrium exists between dimer and monomer, which is dependent on temperature, protein and ionic concentrations (Driessen, 1993; Woodbury et al., 2002; Bu et al., 2003). Recently it was also suggested that the ATPase domain of SecA can form a tetramer in solution (Dempsey et al., 2002). These hydrodynamic properties have been suggested to be important in in vivo translocation of secretory proteins across cytoplasmic membrane (Driessen, 1993; Or et al., 2002). Nevertheless, the critical role for dimerization of SecA during secretion is still obscure. Thus, further investigation would be necessary to reveal effects of SecA oligomerization on its function.

\footnotetext{
*To whom correspondence should be addressed.

Tel: 82-62-530-2823; Fax: 82-62-530-2809

E-mail: thahn@chonnam.ac.kr
} 
In previous work, we reported that the functional signal peptides render SecA form more open structure, thereby facilitating a penetration into membranes with an increase of membrane bound SecA (Ahn and Kim, 1996). Here, we provide evidence that the signal peptide of ribose-binding protein (RBP) stimulates the oligomerization of SecA, and thus increases the binding of SecA to membranes. Our results also indicate that nucleotides induce the dissociation of SecA oligomer into less degree of oligomer, thereby reducing its membrane binding. From these results, we propose that membrane binding of soluble SecA might be regulated by its oligomerization, which is influenced by translocation components as suggested previously (Or et al., 2002; Benach et al., 2003). The mode of interaction between SecA and signal peptide/ATP is also discussed.

\section{Materials and Methods}

Synthesis and purification of peptides. The RBP signal peptides, wild-type (WT), mutant (export-defective mutant peptide, MT), and two revertants (SF, TI), were synthesized and purified as described previously (Yi et al., 1994; Chi et al., 1995). The idealized transmembrane peptide (TM) was chemically synthesized by PepTron Inc. (Daejon, Korea). These peptides (Table 1) were purified by the reverse phase HPLC using a Phenomenex W-porex C8 column $(300 \mathrm{~mm} \times 10 \mathrm{~mm}, 10 \mu \mathrm{m})$. Elution was carried out with a water-acetonitrile linear gradient $(10 \sim 75 \% \quad(\mathrm{v} / \mathrm{v})$ of acetonitrile) containing $0.1 \%(\mathrm{v} / \mathrm{v})$ trifluoroacetic acid. The stock solutions were prepared by dissolving the lyophilized peptides in absolute dimethylsulfoxide. The concentrations of stock solution were determined by quantitative amino acid analysis. Molecular weights of the purified peptide were confirmed using matrix assisted laser desorption ionization (Kratos Kompact MALDI II).

Preparation of SecA and ATPase activity assay. SecA protein was purified from a SecA-overproducing strain (RR1/pMAN400) (Kawasaki et al., 1989) as described (Ahn and Kim, 1996). SecYE(His $\left.{ }_{6}\right) \mathrm{G}$ was expressed in BL21(DE3) cells carrying pBAD22 and purified as described previously (Collinson et al., 2001). The ATPase activity of SecA was assayed by measuring inorganic phosphate $(P i)$ released from ATP as described (Lanzetta et al., 1979). One unit of ATPase activity is defined as the hydrolysis of 1 pmol of ATP per minute.

Preparation of model membranes and proteoliposomes containing SecYEG. Liposomes without SecYEG were prepared as described elsewhere (Ahn and Kim, 1998). Vesicles of mixed lipid composition of $E$. coli phosphatidylethanolamine (PE)/dioleoylphosphatidylglycerol (DOPG) (60:40, by molar ratio) (Avanti Polar Lipids) were used as a standard liposome for model membranes and for reconstitution of SecYEG protein throughout the investigations. To prepared vesicles containing 1-palmitoyl-2-(1-pyrenedecanoyl)-sn-glycero3-phosphoethanolamine (pyrene-PE), this probe was incorporated into liposomes to be $1 \mathrm{~mol} \%$. The phospholipid concentration was determined by phosphorus assay (Vaskovsky et al., 1975). The proteoliposomes containing SecYEG proteins and phospholipids were prepared as described (van der Does et al., 1998).

Effect of signal peptides on the interaction of SecA with ATP. The signal peptide-induced release of ATP from soluble SecA was determined using flow dialysis method as described (Shinkai et al., 1991); ATP $(0.5 \mu \mathrm{M})$ containing $\left[\gamma^{32} \mathrm{P}\right]$ ATP was added to the upper chamber. As indicated by the arrow, $\operatorname{Sec} A(0.4 \mu \mathrm{M})$ was added to the same chamber, followed by adding $4.0 \mu \mathrm{M}$ of signal peptide (WT, SF, TI or MT) after ATP binding to soluble SecA. The inhibition of ATP binding to SecA by signal peptides was carried out in $25 \mathrm{mM}$ Tris-HCl, pH 7.4 containing $100 \mathrm{mM} \mathrm{NaCl}$ and $1 \mathrm{mM}$ dithiothreitol (DTT). SecA $(0.5 \mu \mathrm{M})$ was incubated with 2.0 $\mathrm{mM}$ of ATP containing $1 / 1000$ (by molar ratio) as much $\left[\gamma-{ }^{32} \mathrm{P}\right]$ ATP (Amersham Pharmacia Biotech) in the presence of each indicated concentration of signal peptides for $20 \mathrm{~min}$ at $30^{\circ} \mathrm{C}$. A tenth volume of each sample was filtered through a spun concentrator with molecular weight cutoff of 10,000 . The radioactivity of each filtrate was determined by a liquid scintillation counter (Beckman LS6000LL). The amount of bound ATP to SecA was determined by comparing the radioactivity of filtrate with the control values without protein.

Unfolding and conformational change of soluble SecA. The unfolding of SecA was monitored by measuring intrinsic Trp fluorescence of SecA with Shimadzu RF-5301 PC spectrofluorometer equipped with a thermostated cuvette compartment. All experiments

Table 1. Amino Acid Sequences of the Synthetic Signal Peptides

\begin{tabular}{ll}
\hline \multicolumn{1}{c}{ Peptide } & \multicolumn{1}{c}{ Sequence } \\
\hline WT signal peptide & MNMKKLATLVSAVALSATVSANAMA \\
MT (L9P) $)^{\mathrm{a}}$ & MNMKKLATPVSAVALSATVSANAMA \\
SF (L9P, S11F) & MNMKKLATPVEAVALSATVSANAMA \\
TI $(\text { L9P, T8I })^{\mathrm{b}}$ & MNMKKLAIIPVAVALSATVSANAMA \\
TM $^{\mathrm{c}}$ & KKKKKWALALALALALALALALALALC \\
\hline
\end{tabular}

${ }^{a}$ The precursor RBP with the mutant signal peptide (L9P) was not exported to the periplasmic space.

${ }^{\mathrm{b}}$ The two revertant signal peptides, SF (L9P, S11F) and TI (L9P, T8I), function as intragenic suppressors for the L9P mutant, substantially restoring export of RBP (Song and Park, 1995).

${ }^{c}$ The sequence of the TM peptide is modeled on the transmembrane segments found in the proteins of inner-membrane of $E$. coli (Zheng and Gierasch, 1997). 
were performed with $500 \mu \mathrm{M}$ ATP (or its analog), or $4.0 \mu \mathrm{M}$ of signal peptides in $50 \mu \mathrm{M}$ potassium phosphate buffer, $\mathrm{pH}$ 7.5, containing $0.4 \mu \mathrm{M}$ SecA, $50 \mathrm{mM} \mathrm{NaCl}$, and $1 \mathrm{mM}$ DTT. Before initiating SecA unfolding, SecA solution was pre-incubated with signal peptides or nucleotides at $30^{\circ} \mathrm{C}$ for $20 \mathrm{~min}$. The thermal unfolding of SecA was analyzed by following the decrease in fluorescence intensity at $338 \mathrm{~nm}$ (under excitation wavelength of $298 \mathrm{~nm}$ ) with a $0.5^{\circ} \mathrm{C} / \mathrm{min}$ heating rate. For urea-induced unfolding of SecA, the sample solution was incubated at $30^{\circ} \mathrm{C}$ for $20 \mathrm{~min}$ at each indicated concentration of urea, and then the fluorescence intensities were measured at the same conditions described in the thermal denaturation. The data were fitted to a two-step unfolding model as described (Morjana et al., 1993). The relative Trp fluorescence intensity of SecA was measured as a function of signal peptide or nucleotide concentration at $338 \mathrm{~nm}$ of emission wavelength (under $298 \mathrm{~nm}$ of excitation wavelength).

Oligomerization of SecA. Oligomerization of SecA was investigated by measuring steady-state fluorescence resonance energy transfer between fluorescein 5-maleimide (fluorescein)- and 7-diethylamino3-(4'-maleimidylphenyl)-4-methylcoumarin (coumarin)-labeled SecA molecules as described (Driessen, 1993). Coumarin-labeled SecA was mixed with equal amount of fluorescein-labeled SecA. Samples were exposed at $370 \mathrm{~nm}$, and emission spectra were monitored in the range of 420 to $580 \mathrm{~nm}$ at $30^{\circ} \mathrm{C}$. The fluorescence intensity at $528 \mathrm{~nm}$ was selected as an indicator for the energy transfer. The oligomerization state of SecA was also monitored from fluorescence anisotropy of $\mathrm{Ru}$ bis(2,2'-bipyridine)(2,2'bipyridine-4,4'-dicarboxylic acid) (Ru(bpy $)_{2}(\mathrm{dcbpy})$ ) as described (Terpetschnig et al., 1995; den Blaauwen et al., 1997). Anisotropy was measured using the 8100 Series 2 Spectrofluorometer (SLMAMINCO) equipped with Glan-Thompson calcite prism polarizers in $\mathrm{L}$ format. The emission fluorescence of $\mathrm{Ru}(\mathrm{bpy})_{2}$ (dcbpy) was measured at $650 \mathrm{~nm}$ with an excitation wavelength of $460 \mathrm{~nm}$. The anisotropy values were calculated according to the equation:

$$
A=\left(I_{\mathrm{II}}-I_{\perp}\right) /\left(I_{\mathrm{II}}+2 I_{\perp}\right),
$$

where $I_{\mathrm{II}}$ and $I_{\perp}$ are the intensities measured with polarizers which are parallel and perpendicular to the vertically polarized exciting beam, respectively.

Membrane binding of SecA. Purified SecA was iodinated using IODO-GEN (PIERCE). Free iodine was removed by Sephadex G25 column chromatography. Binding experiments were performed with $0.2 \mu \mathrm{M}{ }^{125}$ I-labeled SecA and $500 \mu \mathrm{M}$ phospholipid (large unilamellar vesicles or proteoliposomes) in the presence of signal peptides (as a function of concentration) or ATP in a buffer containing $50 \mathrm{mM}$ potassium phosphate $(\mathrm{pH} \mathrm{7.4),} 100 \mathrm{mM} \mathrm{KCl}$, and $1 \mathrm{mM}$ DTT. Iodinated SecA was preincubated with signal peptides or nucleotides for $20 \mathrm{~min}$ at $30^{\circ} \mathrm{C}$. Membranes were then added to the reaction mixture and further incubated for $20 \mathrm{~min}$. Protein-bound membranes were collected using Beckman TLA 100.2 rotor at $70,000 \mathrm{rpm}$ for $30 \mathrm{~min}$. The radioactivities of pellets and supernatant were determined using the $\gamma$ counter. The energy transfer between Trp residue(s) in SecA and pyrene-PE incorporated into model membranes was used to measure the insertion of SecA into lipid bilayer as described (Ahn and Kim, 1998).
Effect of signal peptide on the nucleotide binding to membraneassociated SecA. The assay was carried out using the flow dialysis method as described above, except the followings; $0.5 \mathrm{mM}$ ADP or adenosine 5'-[g-thio]triphosphate (ATP $\gamma \mathrm{S})$ each containing 5'-[2,8$\left.{ }^{3} \mathrm{H}\right]$ ADP or $55^{\prime}-\left[\gamma_{-}{ }^{35} \mathrm{~S}\right] \mathrm{ATP}$, respectively. Proteoliposomes $(700 \mathrm{mM}$ of phospholipids and $28 \mathrm{mg}$ of SecYEG) were added to the upper chamber, which was mixed with SecA and/or signal peptide as indicated by arrows (Fig. 7).

Other methods. In vitro translocation and ATPase activity were analyzed with SecA modified with fluorescent probes such as fluorescein, coumarin, and $\mathrm{Ru}(\mathrm{bpy})_{2}$ (dcbpy). In vitro translocation assay was performed with ${ }^{35} \mathrm{~S}$-labeled proOmpA as described (Hartl et al., 1990) due to resistance of ribose-binding protein to proteolysis by protease K. Protein concentration was determined by the Bradford method (Bradford, 1976) using bovine serum albumin as a standard.

\section{Results}

Interaction of signal peptides and ATP with soluble SecA. In order to solve controversies over sequential interaction of soluble SecA with secretory components, especially preprotein and ATP, during early translocation step, we measured release of ATP from the preformed ATP-SecA complex by signal peptides from the ribose-binding protein (Fig. 1A). After $[\gamma-$ ${ }^{32} \mathrm{P}$ ]ATP was added to the upper chamber, the radioactivity in the dialysate abruptly increased and then decreased at a slow rate. When SecA was added to the upper chamber, the radioactivity decreased markedly, indicating the formation of SecA-ATP complex. Subsequent addition of signal peptide immediately increased radioactivity in the dialysate with decreasing order of efficiencies, i.e. $\mathrm{WT}>\mathrm{SF} \geq \mathrm{TI}>\mathrm{MT}$, suggesting that the bound ATP was released by signal peptides. The signal peptide-induced increase of radioactivity does not occur due to the ATPase activity of SecA but due to the release of bound ATP, which is supported by the signal peptide-induced decrease of endogenous ATPase activity of SecA (Fig. 1C). The effect of export-defective peptide (MT) was much lower.

The effect of signal peptides on ATP binding to SecA in an equilibrium state was shown in Fig. 1B. When only SecA was present, the ratio of ATP binding to SecA occurred approximately at a ratio of $1: 1$, which appears to be in the mode of highaffinity binding reported previously (Mitchell and Oliver, 1993). However in the presence of signal peptides, the ATP binding is proportionally reduced with an increase in concentration and functionality of signal peptide: the WT signal peptide shows a reduction of about $75 \%$, i.e. $0.25 \mathrm{~mol}$ of ATP $/ \mathrm{mol}$ of SecA, compared to that of control peptide (TM) with a sequence of $\mathrm{K}_{5} \mathrm{~W}(\mathrm{AL})_{10}$. The TM sequence was reported as an idealized transmembrane peptide (Zheng and Gierasch, 1997). Although this peptide exhibits similar properties to signal peptide, e.g. N-terminal positive charge, 
(A)

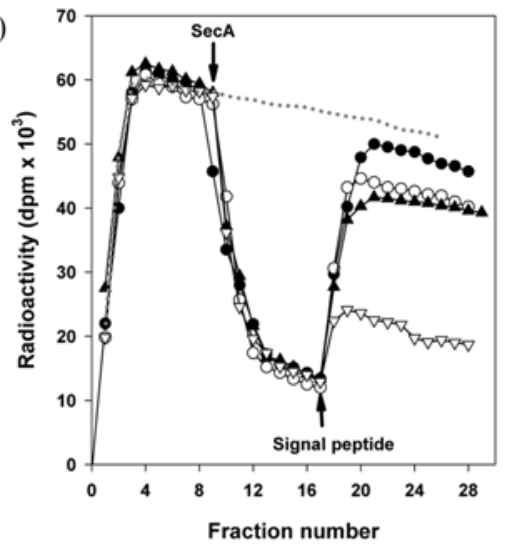

(B)

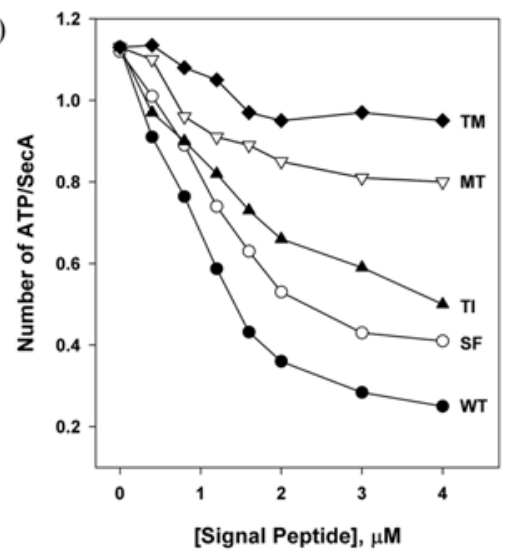

(C)

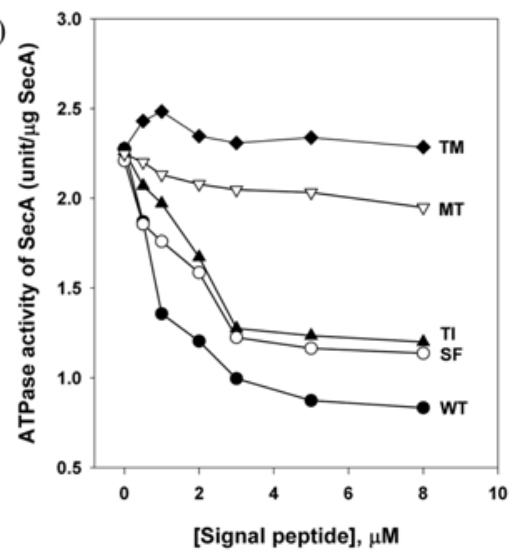

Fig. 1. Effect of signal peptides on the interaction of SecA with ATP. The signal peptide-induced release of ATP from SecA was determined using the flow dialysis method (A). SecA was added to ATP solution, which was mixed with $4.0 \mathrm{mM}$ of signal peptides as indicated by arrows. Each symbol represents WT $(\boldsymbol{O})$, SF $(\bigcirc)$, TI $(\boldsymbol{\Delta})$, and MT $(\nabla)$. The inhibition of ATP binding to SecA by signal peptides was also measured (B) as described in "Materials and Methods". TM represents a transmembrane peptide with a sequence of $\mathrm{K}_{5} \mathrm{~W}(\mathrm{AL})_{10}$. In (C), the endogenous ATPase activity of SecA was analyzed at $30^{\circ} \mathrm{C}$ as a function of signal peptide concentration in the absence of lipid components. One unit of ATPase activity is defined as the hydrolysis of 1 pmol of ATP per minute.
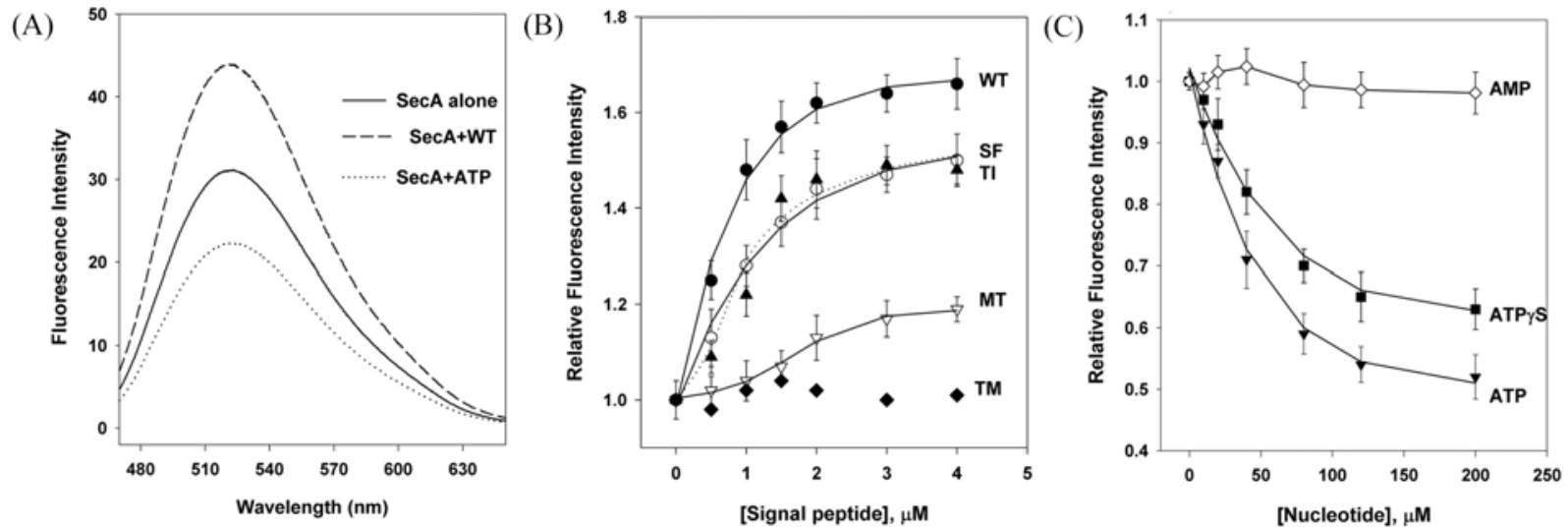

Fig. 2. Resonance energy transfer between fluorescein- and coumarin-labeled SecA. The change in emission fluorescence of fluorescein was measured at $30^{\circ} \mathrm{C}$ for assaying the transferred energy in the presence of $4.0 \mu \mathrm{M}$ signal peptide or $500 \mu \mathrm{M}$ ATP (A). The relative intensity of fluorescence emission was selected at $528 \mathrm{~nm}$ and plotted as a function of signal peptide (B) or nucleotide (C) concentration. SecA $(0.4 \mu \mathrm{M})$ was mixed with each indicated concentration of signal peptides or nucleotides. Data points represent mean \pm S.E. of three independent experiments.

hydrophobicity, and penetration into membranes, it does not significantly influence the ATP binding to or release from SecA. The signal peptides from revertants, SF and TI (Teschke et al., 1991; Song and Park, 1995), showed 50 60\% decrease in bound ATP, whereas the MT had a smaller effect $(<20 \%)$. This indicates that the wild-type and two revertant signal peptides inhibit ATP binding to soluble SecA.

In order to obtain further evidence on the signal peptideinduced inhibition of ATP-binding to and release from SecA, we measured the residual ATPase activity of SecA in the presence of signal peptides. The signal peptides of WT, SF, and TI decreased the ATPase activity far more than the MT or TM peptide (Fig. 1C), which is consistent with the previous report that functional signal peptides inhibit ATPase activity of N-terminal domain of SecA (Triplett et al., 2001). Thus, it is likely that soluble SecA primarily interacts with signal peptides rather than ATP in early translocation pathway. However, the results do not directly imply that signal peptide and ATP bind SecA at the same site in mutually exclusive manner.

Regulation of SecA oligomerization by ATP and signal peptides. In order to assess the functional implication of SecA oligomerization during translocation, we analyzed the effects of ATP, ATP analog and signal peptide on degree of SecA oligomerization using the resonance energy transfer technique (Driessen, 1993). When equal molar concentration of coumarin-labeled SecA was present, a change in fluorescence emission spectra of fluorescein attached to $\operatorname{Sec} \mathrm{A}$ was shown in Fig. 2A. The signal peptides exhibited a concentration- 
(A)

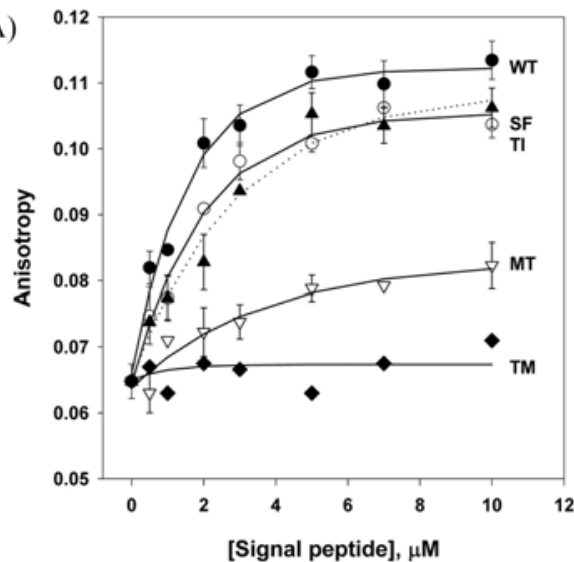

(B)

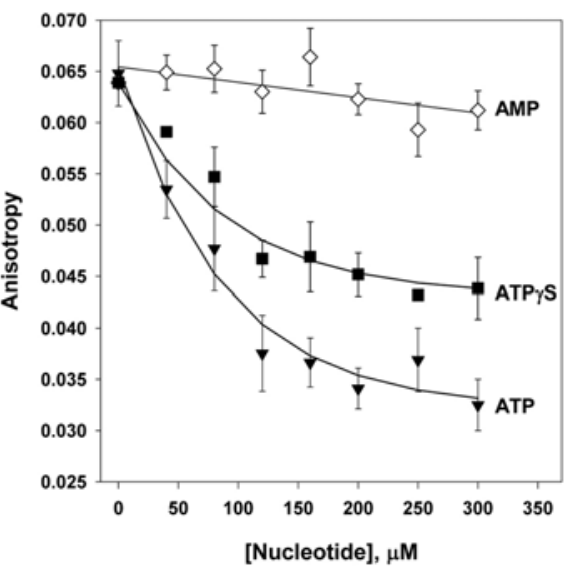

Fig. 3. Steady state fluorescence anisotropy of $\mathrm{Ru}(\mathrm{bpy})_{2}(\mathrm{dcbpy})$-labeled SecA. Native SecA molecules were labeled with $\mathrm{Ru}(\mathrm{bpy})_{2}(\mathrm{dcbpy})$ and the fluorescence anisotropy of soluble $\operatorname{Sec} \mathrm{A}(0.4 \mu \mathrm{M})$ was then determined at $30^{\circ} \mathrm{C}$ in the presence of signal peptides (A) or nucleotides (B) as described in "Materials and Methods". Data points represent mean \pm S.E. of three independent experiments.

dependent increase of energy transfer with the order of $\mathrm{WT}>\mathrm{SF} \approx \mathrm{TI}>\mathrm{MT}$, whereas the TM peptide had no effect (Fig. 2B). The result suggests that signal peptide induce an equilibrium shifts towards the formation of SecA oligomer, which coincides with the recent observation on the signal peptide-dependent oligomerization of SecA (Benach et al., 2003). Our result further demonstrates an importance of in vivo functionality of signal peptides in SecA oligomerization. We observed that increasing concentration of the nucleotides ATP or ATP $\gamma$ S, a non-hydrolysable ATP analog, decreased the energy transfer with varying degree (Fig. 2C). The more drastic effect was observed with ATP than with ATP $\gamma \mathrm{S}$, indicating that these nucleotides induce a shift of oligomeric SecA into monomer and/or lesser degree of oligomer. In contrast, AMP used as a control did not exert any effect, excluding the possibility that the nucleotide effects might be due to quenching or inner filter effects. It is known that AMP has the same spectral properties as ATP but is unable to bind to SecA.

To confirm the energy transfer result (Fig. 2), we measured the fluorescence anisotropy of SecA labeled with $\mathrm{Ru}(\mathrm{bpy})_{2}$ (dcbpy). The rotational correlation coefficient of SecA is in the order of 50-70 ns (den Blaauwen et al., 1997) and the fluorescence life-time of $\mathrm{Ru}(\mathrm{bpy})_{2}$ (dcbpy) is about $400 \mathrm{~ns}$ (Terpetschnig et al., 1995). Thus, the probe seems to be suitable for recording changes in the oligomerization state of SecA. As shown in Fig. 3A, all signal peptides tested here increased the anisotropy in a concentration-dependent manner, indicating that the signal peptides induce a shift towards the higher oligomeric form of SecA compared to that of soluble SecA alone. It was also found that the levels of increases in SecA anisotropy by the wild type and two revertants (SF and TI) are much higher than that of the MT or positive control (TM peptide).

On the other hand, ATP decreased the anisotropy, implying the dissociation of oligomeric SecA upon interaction with the nucleotide. ATP $\gamma \mathrm{S}$ also reduced the anisotropy but with lesser degree. From these results, we conclude that signal peptides and ATP have opposite effects on the dynamic state of SecA. When in vitro translocation and ATPase activity of SecA labeled with fluorescent probes were measured as a control experiment, there were no apparent differences in the activities compared to those of native SecA (results not shown).

Unfolding and conformational change of SecA. In order to relate structural change of SecA to its oligomerization, we observed effects of ATP and signal peptide on temperature- or urea-induced unfolding of $\operatorname{Sec} \mathrm{A}$ as monitored by intrinsic fluorescence of Trp residues in the protein. The SecA protein itself exhibited a two-step unfolding process (native $\rightarrow$ intermediate $\rightarrow$ denatured) induced by urea with an intermediate formed at 1.5 2.6 $\mathrm{M}$ of denaturant (Fig. 4A). However, the presence of ATP slightly shifted concentration of urea for the intermediate formation to $1.8 \sim 3.0 \mathrm{M}$. In thermal unfolding (Fig. 4B), the native SecA had the transition temperature $\left(\mathrm{T}_{\mathrm{m}}\right)$ of $41^{\circ} \mathrm{C}$, which is similar to the one obtained by Ulbrandt et al. (1992). This was increased up to 43.6 and $49.8^{\circ} \mathrm{C}$ in the presence of $\mathrm{ADP}$ and ATP, respectively. Here, the $\mathrm{y}$-axis (fractional change) is relative fluorescence changes with the fluorescence intensity of SecA set to zero (0) and one (1) at $17^{\circ} \mathrm{C}$ and the final temperature, respectively. The emission intensity of native SecA measured at $338 \mathrm{~nm}$ decreases as temperature increases and reaches a steady state at about $50^{\circ} \mathrm{C}$. Although the result does not address the type of SecA structure, it may imply that a more compact (closed) form of SecA is achieved upon interaction with ATP as suggested previously (Morjana et al., 1993; Wang et al., 2000; Hunt et al., 2002).

Table 2 summarizes the values of midpoints from thermal unfolding and the concentrations of urea maintaining the intermediate of SecA. Unlike ATP, the WT signal peptide 
(A)

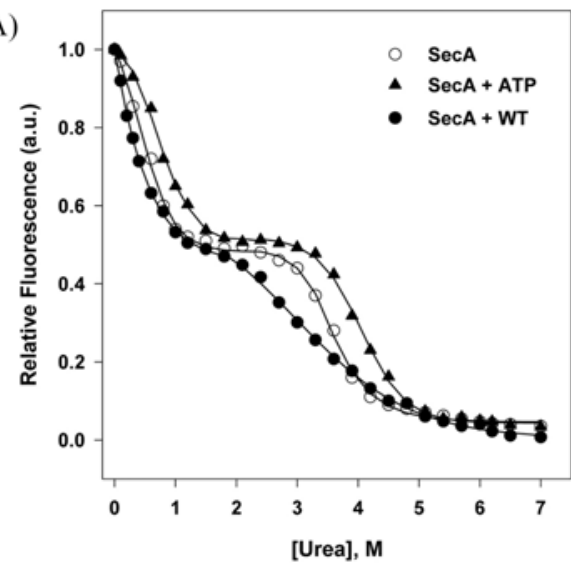

(B)

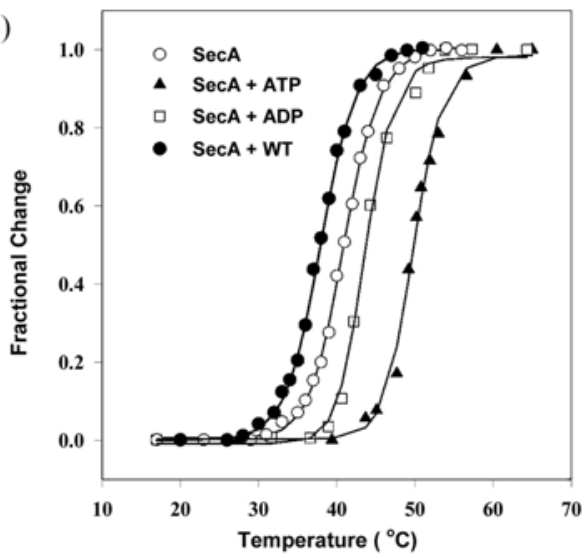

Fig. 4. Effect of signal peptide or ATP on the unfolding of SecA. The urea- (A) or temperature- (B) induced unfolding of SecA was conducted in the presence of signal peptides or nucleotides. Change in the fluorescence intensity at $338 \mathrm{~nm}$ was recorded as a function of temperature or urea concentration. First, signal peptides or nucleotides were mixed with SecA solution and incubated for 20 min at $30^{\circ} \mathrm{C}$. In thermal unfolding, the mixture was initially cooled to $17^{\circ} \mathrm{C}$ before increasing temperature. The concentrations of SecA, signal peptides, and nucleotides were $0.4,4.0$, and $500 \mu \mathrm{M}$, respectively.

Table 2. Midpoint $\left(\mathrm{T}_{\mathrm{m}}\right)$ of the Thermal Denaturation and the Concentrations of Urea Maintaining the Intermediate structure of SecA

\begin{tabular}{ccccc}
\hline \multirow{2}{*}{ Unfolding } & \multicolumn{4}{c}{ Translocation Components } \\
\cline { 2 - 5 } & SecA alone & + WT peptide & + ATP & + ADP \\
\hline Thermally-induced $\left({ }^{\circ} \mathbf{C}\right)$ & $40.9 \pm 0.3$ & $37.4 \pm 0.5$ & $49.8 \pm 0.4$ & $43.6 \pm 0.3$ \\
Urea-induced $(M)$ & $1.5 \sim 2.6$ & - & $1.8 \sim 3.1$ & $1.6{ }^{\circ} 2.8$ \\
\hline
\end{tabular}

exerted a positive effect on SecA unfolding; $\mathrm{T}_{\mathrm{m}}$ of the thermal unfolding became lower than that of SecA alone, and the intermediate in urea-induced unfolding disappeared. This result indicates that $\mathrm{Sec} A$ assumes a more open structure upon interaction with the signal peptide. When the urea-induced unfolding of SecA was carried out at 10 or $20^{\circ} \mathrm{C}$, the intermediate was not detectable, and thus the denaturation curve becomes a one-step process (results not shown) as observed by Doyle et al. (2000). On the contrary, the unfolding process at $30^{\circ} \mathrm{C}$ follows the two-step model.

The conformational change of SecA was also examined using fluorescence in the presence of signal peptide or nucleotide. As shown in Fig. 5B, ATP decreased the fluorescence intensity of SecA by $25 \%$ without any shift in the maximum wavelength $\left(\lambda_{\max }\right)$ and ATP $\gamma \mathrm{S}$ showed lesser effect $(17 \%$ decrease at $200 \mathrm{mM})$. In contrast, the signal peptides, WT, SF, and TI, increased fluorescence by $27 \sim 45 \%$ compared to that of SecA alone without changing $\lambda_{\max }$ (Fig. $5 \mathrm{~A})$. These observations clearly suggest that ATP and signal peptide bring about the opposite effects on the SecA conformation. Thus, it is tempting to speculate that the degree of SecA oligomerization is coupled to ATP or signal peptideinduced structural changes of the protein.

Effect of signal peptides or ATP on membrane binding of SecA. In order to examine whether signal peptide or ATPinduced change of SecA oligomerization has an influence on its membrane binding, we conducted an assay for measuring amounts of SecA associated with SecYEG-reconstituted proteoliposomes. In this experiment, soluble SecA was preincubated with signal peptide or nucleotide prior to incubation with proteoliposomes. The signal peptides increased the SecA binding to membranes with different efficiencies (WT $>\mathrm{SF} \geq$ TI $>$ MT, Fig. 6A). The effect of mutant peptide was only marginal. In contrast, $\mathrm{ATP}$ and $\mathrm{ATP} \gamma \mathrm{S}$ reduced the amounts of membrane bound SecA (Fig. 6B). We obtained similar result with the model membranes consisting of phospholipids without SecYEG complex (results not shown). The reduced binding and/or insertion of SecA into bilayer by nucleotides was also confirmed by measuring resonance energy transfer between SecA Trp residue(s) and pyrene-PE incorporated into model membranes (Fig. 6B, inset). The pyrene group is attached to the end of decanoyl chain of $s n-2$ position, while SecA has seven Trp residues distributed throughout its sequence. $F / F_{o}$ values were increased with increasing concentrations of nucleotides, indicating a reduction of energy transfer efficiency. A control experiment was carried out in the absence of vesicles and analyzed after the amount of pelleted SecA was subtracted.

It was shown that ATP was released from soluble SecA by the signal peptides in Fig. 1. Here, we investigated the interaction of SecA with nucleotides when the protein was associated with the membrane. As a result, all signal peptides tested increased the nucleotide binding activity of SecA that 

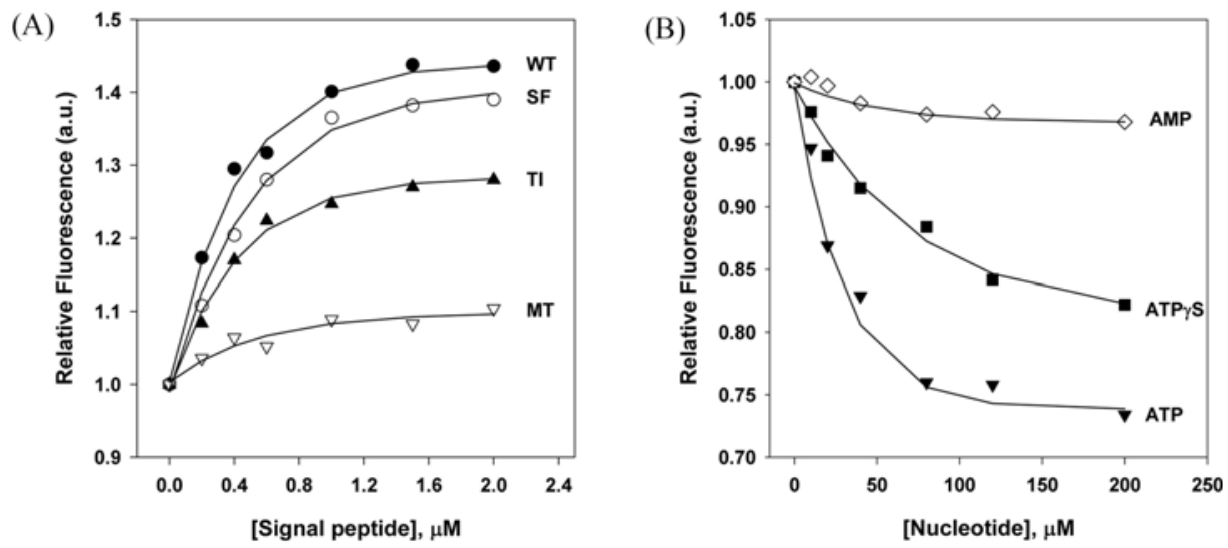

Fig. 5. The intrinsic Trp fluorescence change of SecA. Relative Trp fluorescence intensity was measured as a function of signal peptide (A) or nucleotide (B) concentration. The fluorescence intensity of $0.4 \mu \mathrm{M} \mathrm{SecA}$ at $338 \mathrm{~nm}$ was recorded in the presence of indicated concentration of signal peptides or nucleotides at $30^{\circ} \mathrm{C}$.
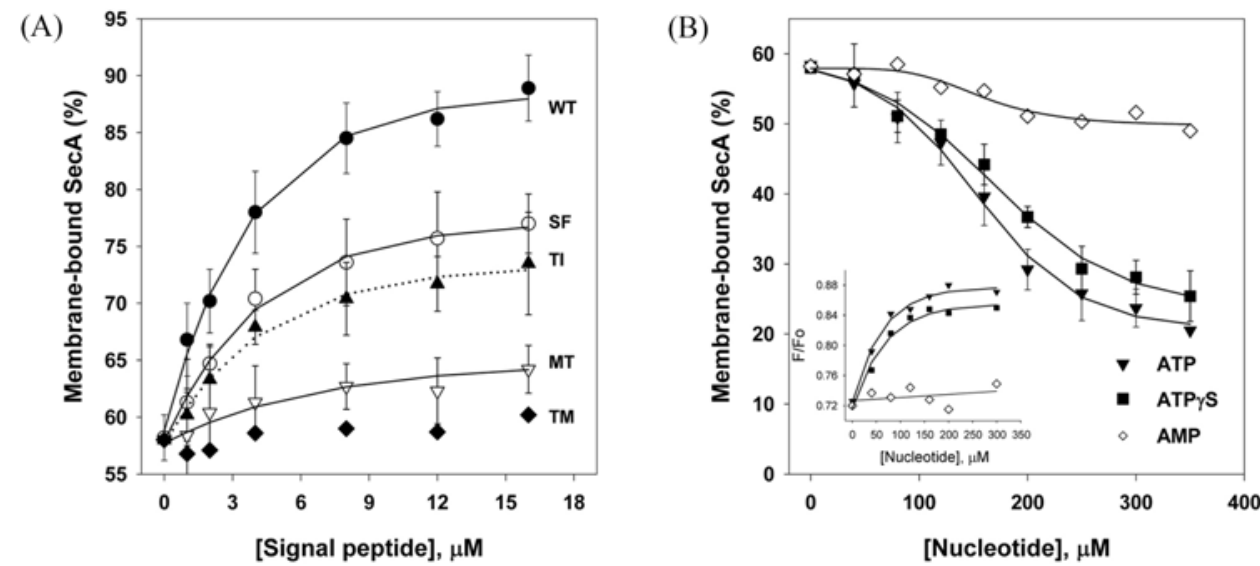

Fig. 6. Signal peptide or nucleotide-dependent binding of SecA to proteoliposomes containing SecYEG Binding of SecA to membranes was measured in the presence of signal peptides (A) or nucleotides (B) as described in "Materials and Methods". ${ }^{125} \mathrm{I}$-labeled SecA was preincubated with each indicated concentration of signal peptides or nucleotides for $20 \mathrm{~min}$ at $30^{\circ} \mathrm{C}$ and then followed by adding membranes. Vesicles with bound SecA were pelleted by ultracentrifugation (in Beckman TLA 100.2 rotor at 70,000 rpm for $30 \mathrm{~min}$ ), and the radioactivities of pellets and supernatants were determined by $\gamma$-ray counting. Inset shows resonance energy transfer between Trp residue(s) of SecA $(0.4 \mu \mathrm{M})$ and pyrene-PE incorporated into membranes consisting of phospholipids $(700 \mu \mathrm{M})$ without SecYEG. $\mathrm{F} / \mathrm{F}_{\mathrm{o}}$ represents the fluorescence intensity ratio at $338 \mathrm{~nm}$ for the sample with $(\mathrm{F})$ and without $\left(\mathrm{F}_{\mathrm{o}}\right)$ pyrene-PE.

was associated with proteoliposomes in a concentrationdependent manner (Fig. 7). The WT signal peptide exerted the highest effect, and the two revertants, SF and TI, with lower effects. Even the mutant peptide, MT, slightly enhanced the association of membrane-bound SecA with ATP $\gamma \mathrm{S}$, although the level was clearly below those of wild-type and revertant signal peptides. The proteoliposome and/or signal peptide itself did not show any binding activity for nucleotide tested here (results not shown). We obtained essentially the same result as Fig. 7 (result not shown) when we added SecA protein and signal peptide to the upper chamber of flow dialysis system (as described in "Materials and Methods"), containing proteoliposomes, and incubated $30 \mathrm{~min}$ prior to the addition of nucleotides, which may allow formation of fully membrane-bound SecA. This provides indirect evidence that membrane-bound SecA with signal peptide resume its nucleotide binding activity. The result is consistent with the finding that signal peptides stimulate ATPase activity of SecA in the absence of preprotein (Miller et al., 1998; Wang et al., 2000).

\section{Discussion}

SecA interacts with various components during translocation, leading to conformational changes and concomitant alteration of binding property to membrane (Ulbrandt et al., 1992; Ding et al., 2001). Recently, the regulation of SecA oligomerization through interaction with translocation components such as phospholipids, signal peptides, and nucleotides has been 
(A)

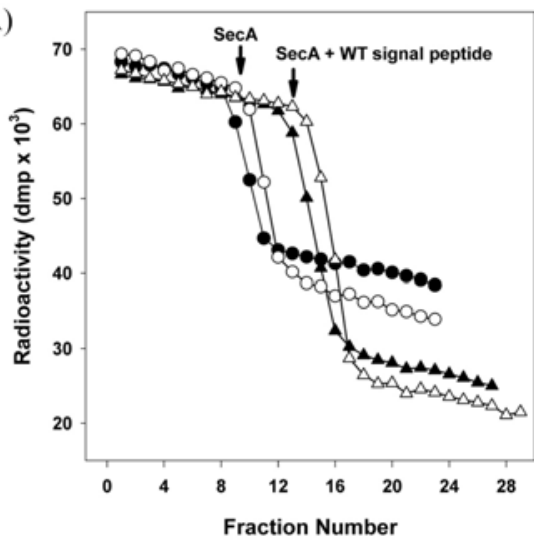

(B)

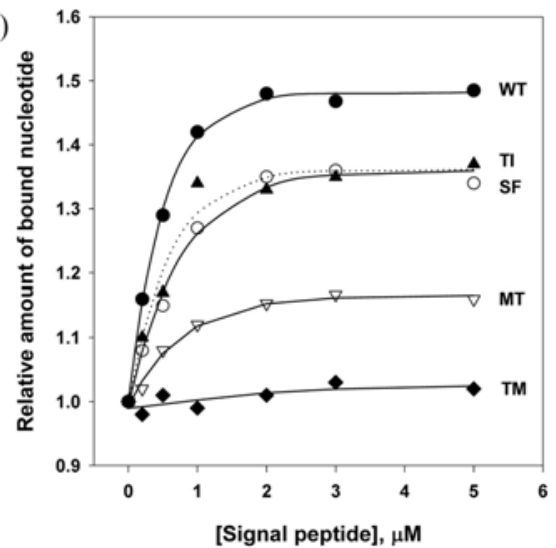

Fig. 7. Effect of signal peptides on the nucleotide binding activity of SecA associated with membranes containing SecYEG. (A) The assay was carried out as described in "Materials and Methods". Nucleotides $(0.5 \mu \mathrm{M})$ containing $5{ }^{\prime}-\left[\gamma_{-}{ }^{35} \mathrm{~S}\right] \mathrm{ATP}$ or 5 '-[2,8- $\left.{ }^{3} \mathrm{H}\right] \mathrm{ADP}$ and proteoliposomes were added to the upper chamber. As indicated by the arrows, $0.4 \mu \mathrm{M}$ of SecA $(\mathbf{O}, \bigcirc)$ or SecA plus $4.0 \mu \mathrm{M}$ of wildtype (WT) signal peptide $(\boldsymbol{\Delta}, \triangle)$ were added to the upper chamber. Closed and open symbols represent the binding of ATP $\gamma \mathrm{S}$ and ADP to SecA, respectively. In (B), the relative amount of ATP $\gamma \mathrm{S}$ bound to SecA was plotted as a function of signal peptide concentration, based on result obtained from flow dialysis method. The amount of ATP $\gamma$ S bound to SecA in the absence of signal peptide was assumed as one value in y-axis.

implicated in the secretion process (Or et al., 2002; Benach et al., 2003). In the present work, we observed differential effects of signal peptide and ATP on the oligomerization and membrane binding of SecA. The signal peptide induced an increase in SecA oligomerization, while ATP stimulated the dissociation of oligomeric form of the protein. As indicated by SecA unfolding, the degree of oligomerization seems to be associated with the formation of open or closed structure of the protein that is inducible by an interaction with signal peptide or nucleotide, respectively. We suggest that oligomeric form of SecA increases the binding to membranes, and on the contrary, the dissociation of SecA oligomer reduces the membrane association. The in vivo functionality of signal peptides was shown to be critical in the oligomerization and membrane binding of SecA as well as in inhibition of ATP binding to soluble SecA. However, the present results do not imply that the oligomeric form of SecA is stabilized in the membrane and that it is the native form for SecA function in vivo. Rather, membrane or phospholipid has been suggested to induce an equilibrium shift to the monomeric form of SecA (Or et al., 2002; Benach et al., 2003).

The ATP-induced inhibition of SecA binding to membranes seems to contradict with previous results in which the energy for ATP binding drives membrane insertion of a $30 \mathrm{kDa}$ domain of SecA, while dissociation of SecA requires the hydrolysis of ATP (Economou and Wickner, 1994; Economou et al., 1995). At present, we do not provide a satisfactory explanation for this discrepancy. It may possibly reflect differences in the behavior of SecA-signal peptide (or preprotein) complex, which is dependent on the presence or absence of membranes: the ATP-driven membrane insertion of SecA has been observed in the presence of preprotein. Actually, we found that the presence of signal peptide make
SecA penetrate deeper into membrane bilayers and traverse it through interacting with ATP, which was inferred from the protection of SecA domains from cytosolic protease digestion (Economou and Wickner, 1994; Ahn and Kim, 1996) as well as from the fragmentation of SecA by protease encapsulated within vesicles (Ahn and Kim, 1994) (results not shown). Thus, we speculate that ATP plays different roles in conformational changes of soluble and membrane-associated SecA and in subsequent events for membrane binding/ insertion.

The decrease in anisotropy by nucleotides also conflicts with the results of den Blaauwen et al. (1997). They observed an increase in rotational correlation time from 50 ns to 82 ns with ATP, which is different from the results reported here. This discrepancy might be due to differences in experimental conditions such as concentrations of SecA, salt, and nucleotides. Above all, it is likely that magnesium ion caused a difference in SecA behavior. den Blaauwen et al. (1997) reported that an addition of $5 \mathrm{mM} \mathrm{MgCl} 2$ without nucleotide increases a rotational correlation time of SecA by about $40 \%$ compared to that of magnesium-free SecA. Recently, the complex effect of $\mathrm{Mg}^{2+}$ was also reported in nucleotide-dependent SecYEGSecA interactions (Duong, 2003). In that paper, ATP or $\mathrm{ATP} \gamma \mathrm{S}$ induced dissociation of SecA oligomer from SecYEG complex and increased the interaction of SecYEG with SecA monomer. But the effect of ATP or ATP $\gamma \mathrm{S}$ was strongly reduced when magnesium was included in the reaction buffer. In order to avoid unexpected effects of $\mathrm{Mg}^{2+}$ on SecA oligomerization, we used $\mathrm{Mg}^{2+}$ concentration of less than 0.5 $\mathrm{mM}$. SecA concentration and temperature might also be important in assaying monomer-oligomer equilibrium of the protein (Benach et al., 2003). Indeed, the increase of rotational correlation time of $\operatorname{Sec} \mathrm{A}$ was not found at relatively 
higher concentration of $\operatorname{Sec} \mathrm{A}(0.4 \mathrm{mM})$ and lower $\mathrm{MgCl}_{2}(0.3$ $\mathrm{mM})$ at $30^{\circ} \mathrm{C}$ compared to the conditions used by den Blaauwen et al. (1997) (results not shown).

Regarding dynamics of SecA, the higher oligomeric state (tetramer) was recently suggested (Bu et al., 2003). However, the present results obtained with the energy transfer and fluorescence anisotropy, did not clearly establish whether oligomeric form of SecA is a dimer, tetramer, and/or a higher order form. Also, the exact structural change of SecA interacting with signal peptide or nucleotide was not detected. Therefore, direct correlation between the stability and the oligomerization of SecA could not be made. Nonetheless, we were able to deduce the following relationship involving SecA; the conformational change induced by SecA plus signal peptide or nucleotide is responsible for dimerization and membrane binding of SecA.

It was demonstrated that the N-terminal domain of SecA showed specificity to SecYEG-binding activity (Dapic and Oliver, 2000), while the C-domain was for phospholipid binding (Breukink, et al., 1995). At present, we do not have an evidence on which region(s) of SecA undergoes a conformational change upon interaction with nucleotide or signal peptide. However, based on the fact that both signal peptide and ATP influence the binding of SecA to model membranes and proteoliposomes with SecYEG, we speculate that both $\mathrm{N}$ - and C-domains of SecA are altered by the translocation components. Indeed, Ding et al. (2001) suggested that signal peptide induces a conformational change in the C-domain of SecA.

Conclusively, we were able to envision the following scenario for sequential event of early secretion process involved with SecA. The soluble SecA exists as an ATP-bound form until the signal peptide emerges. Here, conformational equilibrium of SecA molecules is shifted towards monomeric state with suppressed membrane-binding or insertion activity. However, as the signal peptide becomes recognized by SecA, the bound ATP is released from $\operatorname{Sec} A$ and replaced by signal peptide. Signal peptide increases the amount of oligomeric SecA, and at the same time induces an open conformation. The resulting SecA-signal peptide complex then associates with membranes, resuming ATP binding activity.

Acknowledgements This work was supported by a Korea Research Foundation Grant (KRF-2004-005-E00015).

\section{References}

Ahn, T. and Kim, H. (1994) SecA of Escherichia coli traverses lipid bilayer of phospholipid vesicles. Biochem. Biophys. Res. Comm. 203, 326-330.

Ahn, T. and Kim, H. (1996) Differential effect of precursor ribose binding protein of Escherichia coli and its signal peptide on the SecA penetration of lipid bilayer. J. Biol. Chem. 271, $12372-12379$

Ahn, T. and Kim, H. (1998) Effects of nonlamellar-prone lipids on the ATPase activity of SecA bound to model membranes. $J$. Biol. Chem. 273, 21692-21698.

Benach, J., Chou, Y.-T., Fak, J. J., Itkin, A., Nicolae, D. D., Smith, P. C., Wittrock, G., Floyd, D. L., Golsaz, C. M., Gierasch, L. M. and Hunt, J. F. (2003) Phospholipid-induced monomerization and signal-peptide-induced oligomerization of SecA. J. Biol. Chem. 278, 3628-3638.

Bradford, M. M. (1976) A rapid and sensitive method for the quantitation of microgram quantities of protein utilizing the principle of protein-dye binding. Anal. Biochem. 72, 248-254.

Breukink, E., Nouwen, N., van Raalte, A., Mizushima, S., Tommassen, J. and de Kruijff, B. (1995) The C terminus of SecA is involved in both lipid binding and SecB binding. $J$. Biol. Chem. 270, 7902-7907.

Breukink, E., Demel, R. A., de Korte-Kool, G. and de Kruijff, B. (1992) SecA insertion into phospholipid is stimulated by negatively charged lipids and inhibited by ATP: a monolayer study. Biochemistry 31, 1119-1124.

Brundage, L., Hendrick, J. P., Schiebel, E., Driessen, A. J. M. and Wickner, W. (1990) The purified E. coli integral membrane protein SecY/E is sufficient for reconstitution of SecAdependent precursor protein translocation. Cell 62, 649-657.

Bu, Z., Wang, L. and Kendall, D. A. (2003) Nucleotide binding induces changes in the oligomeric state and conformation of SecA in a lipid environment: a small-angle neutron-scattering study. J. Mol. Biol. 332, 23-30.

Cabelli, R. J., Dolan, K. M., Qian, L. and Oliver, D. B. (1991) Characterization of membrane-associated and soluble states of SecA protein from wild-type and SecA51(TS) mutant strains of Escherichia coli. J. Biol. Chem. 266, 24420-24427.

Chi, S.-W., Yi, G.-S., Suh, J.-Y., Choi, B.-S. and Kim, H. (1995) Structures of revertant signal sequences of Escherichia coli ribose binding protein. Biophys. J. 69, 2703-2709.

Collinson, I., Breyton, C., Duong, F., Tziatzios, C., Schubert, D., Or, E., Rapoport, T. and Kuhlbrandt, W. (2001) Projection structure and oligomeric properties of a bacterial core protein translocase. EMBO J. 20, 2462-2471.

Dapic, V. and Oliver, D. (2000) Distinct membrane binding properties of $\mathrm{N}$ - and C-terminal domains of Escherichia coli SecA ATPase. J. Biol. Chem. 275, 25000-25007.

Dempsey, B. R., Economou, A., Dunn, S. D. and Shilton, B. H. (2002) The ATPase domain of SecA can form a tetramer in solution. J. Mol. Biol. 315, 831-843.

den Blaauwen, T., Terpetschnig, E., Lakowicz, J. R. and Driessen, A. J. M. (1997) Interaction of SecB with soluble SecA. FEBS Lett. 416, 35-38.

Ding, H., Mukerji, I. and Oliver, D. (2001) Lipid and signal peptide-induced conformational changes within the C-domain of Escherichia coli SecA protein. Biochemistry 40, 1835-1843.

Douville, K., Price, A., Eichler, J., Economou, A. and Wickner, W. (1995) SecYEG and SecA are the stoichiometric components of preprotein translocase. J. Biol. Chem. 270, 20106-20116.

Doyle, S. M., Braswell, E. H. and Teschke, C. M. (2000) SecA folds via a dimeric intermediate. Biochemistry 39, 1166711676.

Driessen, A. J. (1993) SecA, the peripheral subunit of the Escherichia coli precursor protein translocase, is functional as a dimer. Biochemistry 32, 13190-13197.

Duong, F. (2003) Binding, activation and dissociation of the 
dimeric SecA ATPase at the dimeric SecYEG translocase. EMBO J. 22, 4375-4384.

Economou, A., Pogliano, J. A., Beckwith, J., Oliver, D. B. and Wickner, W. (1995) SecA membrane cycling at SecYEG is driven by distinct ATP binding and hydrolysis events and is regulated by SecD and SecF. Cell 83, 1171-1181.

Economou, A. and Wickner, W. (1994) SecA promotes preprotein translocation by undergoing ATP-driven cycles of membrane insertion and deinsertion. Cell 78, 835-843.

Hartl, F. U., Lecker, S., Schiebel, E., Hendrick, J. P. and Wickner, W. (1990) The binding cascade of SecB to SecA to SecY/E mediates preprotein targeting to the $E$. coli plasma membrane. Cell 63, 269-279.

Hunt, J. F., Weinkauf, S., Henry, L., Fak, J. J., McNicholas, P., Oliver, D. B. and Deisenhofer, J. (2002) Nucleotide control of interdomain interactions in the conformational reaction cycle of SecA. Science 297, 2018-2026.

Kawasaki, H., Matsuyama, S., Sasaki, S., Akita, M. and Mizushima, S. (1989) SecA protein is directly involved in protein secretion in Escherichia coli. FEBS Lett. 242, 431-434.

Keller, R. C., Snel, M. M., de Kruijff, B. and Marsh, D. (1995) SecA restricts, in a nucleotide-dependent manner, acyl chain mobility up to the center of a phospholipid bilayer. FEBS Lett. 358, 251-254.

Lanzetta, P. A., Alvarez, L. J., Reinach, P. S. and Candia, O. A. (1979) An improved assay for nanomole amounts of inorganic phosphate. Anal. Biochem. 100, 95-97.

Lill, R., Cunningham, K., Brundage, L. A., Ito, K., Oliver, D. and Wickner, W. (1989) SecA protein hydrolyzes ATP and is an essential component of the protein translocation ATPase of Escherichia coli. EMBO J. 8, 961-966.

Manting, E. H. and Driessen, A. J. M. (2000) Escherichia coli translocase: the unraveling of a molecular machine. Mol. Microbiol. 37, 226-238.

Miller, A., Wang, L. and Kendall, D. A. (1998) Synthetic signal peptides specifically recognize SecA and stimulate ATPase activity in the absence of preprotein. J. Biol. Chem. 273, 11409-11412.

Mitchell, C. and Oliver, D. (1993) Two distinct ATP-binding domains are needed to promote protein export by Escherichia coli SecA ATPase. Mol. Microbiol. 10, 483-497.

Morjana, N. A., McKeone, B. J. and Gilbert, H. F. (1993) Guanidine hydrochloride stabilization of a partially unfolded intermediate during the reversible denaturation of protein disulfide isomerase. Proc. Natl. Acad. Sci. USA 90, 2107-2111.

Mori, H. and Ito, K. (2001) The Sec protein-translocation pathway. Trends Microbiol. 9, 494-500.

Or, E., Navon, A. and Rapoport, T. (2002) Dissociation of the dimeric SecA ATPase during protein translocation across the bacterial membrane. EMBO J. 21, 4470-4479.
Shinkai, A., Mei, L. H., Tokuda, H. and Mizushima, S. (1991) The conformation of SecA, as revealed by its protease sensitivity, is altered upon interaction with ATP, presecretory proteins, everted membrane vesicles, and phospholipids. $J$. Biol. Chem. 266, 5827-5833.

Song, T. and Park, C. (1995) Effect of folding on the export of ribose-binding protein studied with the genetically isolated suppressors for the signal sequence mutation. J. Mol. Biol. 253, 304-312.

Terpetschnig, E., Szmacinski, H., Malak, H. and Lakowicz, J. R. (1995) Metal-ligand complexes as a new class of long-lived fluorophores for protein hydrodynamics. Biophys. J. 68, 342350.

Terpetschnig, E., Szmacinski, H. and Lakowicz, J. R. (1995) Fluorescence polarization immunoassay of a high-molecularweight antigen based on a long-lifetime Ru-ligand complex. Anal. Biochem. 227, 140-147.

Teschke, C. M., Kim, J., Song, T., Park, S., Park, C. and Randall, L. L. (1991) Mutations that affect the folding of ribose-binding protein selected as suppressors of a defect in export in Escherichia coli. J. Biol. Chem. 266, 11789-11796.

Triplett, T. L., Sgrignoli, A. R., Gao, F.-B., Yang, Y.-B., Tai, P. C. and Gierasch, L. M. (2001) Functional signal peptides bind a soluble N-terminal fragment of SecA and inhibit its ATPase activity. J. Biol. Chem. 276, 19648-19655.

Ulbrandt, N. D., London, E. and Oliver, D. B. (1992) Deep penetration of a portion of Escherichia coli SecA protein into model membranes is promoted by anionic phospholipids and by partial unfolding. J. Biol. Chem. 267, 15184-15192.

van der Does, C., Manting, E. H., Kaufmann, A., Lutz, M. and Driessen, A. J. M. (1998) Interaction between SecA and SecYEG in micellar solution and formation of the membraneinserted state. Biochemistry 37, 201-210.

Vaskovsky, V. E., Kostetsky, E. Y. and Vasendin, I. M. (1975) A universal reagent for phospholipid analysis. J. Chromatogr. 114, 129-141.

Yi, G.-S., Choi, B.-S. and Kim, H. (1994) Structures of wild-type and mutant signal sequences of Escherichia coli ribose binding protein. Biophys. J. 66, 1604-1611.

Wang, L., Miller, A. and Kendall, D. A. (2000) Signal peptide determinants of SecA binding and stimulation of ATPase activity. J. Biol. Chem. 275, 10154-10159.

Woodbury, R. L., Hardy, S. J. and Randall, L. L. (2002) Complex behavior in solution of homodimeric SecA. Protein Sci. 11, 875-882.

Zheng, N. and Gierasch, L. M. (1997) Domain interactions in E. coli SRP: stabilization of $\mathrm{M}$ domain by RNA is required for effective signal sequence modulation of NG domain. Mol. Cell 1, 79-87. 